\title{
Enumeration of Cylindric Plane Partitions
}

\author{
Robin Langer $\|^{\#}$ \\ ${ }^{1}$ CNRS and LIAFA, Université Paris Diderot - Paris 7, Case 7014, 75205 Paris Cedex 13
}

Résumé. Cylindric plane partitions may be thought of as a natural generalization of reverse plane partitions. A generating series for the enumeration of cylindric plane partitions was recently given by Borodin. As in the reverse plane partition case, the right hand side of this identity admits a simple factorization form in terms of the "hook lengths" of the individual boxes in the underlying shape. The first result of this paper is a new bijective proof of Borodin's identity which makes use of Fomin's growth diagram framework for generalized RSK correspondences. The second result of this paper is a $(q, t)$-analog of Borodin's identity which extends previous work by Okada in the reverse plane partition case. The third result of this paper is an explicit combinatorial interpreation of the Macdonald weight occuring in the $(q, t)$-analog in terms of the non-intersecting lattice path model for cylindric plane partitions.

Résumé. Les partitions planes cylindriques sont une généralisation naturelle des partitions planes renversées. Une série géneratrice pour l'énumeration des partitions planes cylindriques a été donnée récemment par Borodin. Comme dans le cas des partitions planes renversées, la partie droite de cette identité peut être factoriser en terme de "longueur d'équerres" des carrés dans la forme sous-jacente. Le premier résultat de cet article est une nouvelle preuve bijective de l'identité de Borodin qui utilise le cadre de "diagramme de croissance" de Fomin pour la correspondence de RSK géneralisée. Le deuxieme résultat de cette article est une $(q, t)$-déformation d'identité de Borodin qui géneralise un résultat de Okada dans le cas des partitions planes renversées. Le troisième résultat de cet article est une formule combinatoire explicite pour le poids de Macdonald qui utilise le modèle des chemins non-intersectant pour les partitions planes cylindriques.

Keywords: cylindric plane partitions, Macdonald polynomials, Pieri rule, vertex operators, non-intersecting lattice paths, growth diagrams, local rules, generalized RSK correspondences.

\section{Introduction}

Cylindric plane partitions were first introduced by Gessel and Krattenthaler [GK97]. For any binary string $\pi$ of length $T$, a cylindric plane partition with profile $\pi$ may be defined as a sequence of integer partitions:

$$
\left(\mu^{0}, \mu^{1}, \ldots \mu^{T}\right) \quad \mu^{0}=\mu^{T}
$$

such that if $\pi_{k}=1$ then $\mu^{k} / \mu^{k-1}$ is a horizontal strip. Otherwise if $\pi_{k}=0$ then $\mu^{k-1} / \mu^{k}$ is a horizontal strip. The weight of a cylindric partition is given by $|\mathfrak{c}|=\left|\mu_{1}\right|+\left|\mu_{2}\right|+\cdots\left|\mu_{T}\right|$. In the special case where $\mu_{0}=\mu_{T}=\emptyset$ we recover the usual definition of a reverse plane partition [Ada08]. If, in addition to this there are no inversions in the profile, we have a regular plane partition.

\footnotetext{
${ }^{\dagger}$ This work is supported by the ANR grant ANR-08-JCJC-0011 
For those readers who are more familliar with the definition of a plane partition as an array of integers which is weakly decreasing along both rows and columns, the bijection with the "interlacing sequence" model is obtained by reading along the main diagonals. For example:

$$
\begin{aligned}
& \begin{array}{|l|l|l|}
\hline 3 & 3 & 2 \\
\hline & 2 & 1 \\
\hline
\end{array} \\
& \begin{array}{|l|l|l|}
\hline 3 & 2 & 1 \\
\hline 1 & 1 & 1 \\
\hline
\end{array} \\
& \mathfrak{c}=(\emptyset,(1),(3,1),(3,2,1),(3,1),(2), \emptyset)
\end{aligned}
$$

A regular plane partition may also be thought of as a pair of semi-standard young tableau of the same shape. In the case of our example, the two tableaux are:

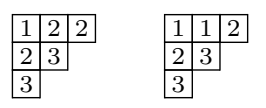

The theory of plane partitions is closely related to both the theory of symmetric functions and Fomin's theory of generalized RSK type correspondences [Fom86, Fom95]. The beginning of the subject is perhaps the following famous identity of MacMahon:

$$
\sum_{\mathfrak{c} \in \mathrm{PP}} z^{|\mathfrak{c}|}=\left(\frac{1}{1-z^{n}}\right)^{n}
$$

It was first pointed out by Okounkov [OR03] that enumerative results for plane partitions may be obtained by considering commutation relations between vertex operators acting on fermionic fock space. The underlying algebraic structure is that of the Heisenberg algebra. By the boson-fermion correspondence these operators may be alternatively thought of as acting on symmetric functions. The Pieri rules for Schur functions are key to this approach:

$$
\begin{aligned}
& S_{\mu}[X] h_{r}[X]=\sum_{\lambda \in U_{r}(\mu)} S_{\lambda}[X] \\
& S_{\lambda}[X+z]=\sum_{\mu \in D_{r}(\mu)} S_{\mu}[X] z^{r}
\end{aligned}
$$

Here $U_{r}(\mu)$ denotes the set of all partitions which can be obtained from $\mu$ by adding a horrizontal $r$-strip and $D_{r}(\lambda)$ denotes the set of all partitions which can be obtained from $\lambda$ by removing a horizontal $r$-strip.

The next important result in the subject is the following hook-product formula for the enumeration of reverse plane partitions with arbitrary profile $\pi$ which is due to Stanley:

$$
\sum_{\mathfrak{c} \in \operatorname{RPP}(\pi)} z^{|\mathfrak{c}|}=\prod_{\substack{i<j \\ \pi_{i}>\pi_{j}}} \frac{1}{1-z^{j-i}}
$$

There is a natural bijection between the boxes of a partition and the inversions in its profile. The expression $j-i$ on the right hand side of Stanley's identity may be understood as the hook length of the corresponding box (see section 2). 
In fact, the right hand side of Stanley's identity may be interpreted as a weighted sum over arbitrarily labelled young diagrams. The weight of such a labelled diagram is equal to a sum over the boxes of the diagram, of the label of the box, times the hook length of the box. The Hillman-Grassl algorithm provides a bijective proof of Stanley's identity.

More recently the following hook-product formula for the enumeration of cylindric plane partitions of given profile was first given by Borodin [Bor07]. A very different proof involving the representation theory of $\widehat{s l}(n)$ was later given by Tingley [Tin08]:

$$
\sum_{\mathfrak{c} \in \operatorname{CPP}(\pi)} z^{|\mathfrak{c}|}=\prod_{n \geq 0}\left(\frac{1}{1-z^{n T}} \prod_{\substack{i<j \\ \pi_{i}>\pi_{j}}} \frac{1}{1-z^{j-i+n T}} \prod_{\substack{i>j \\ \pi_{i}>\pi_{j}}} \frac{1}{1-z^{j-i+(n+1) T}}\right)
$$

Here $T$ denotes the length of the profile. As in the reverse plane partition case, there is a natural bijection between the "boxes" of the cylindric plane partition and "cylindric inversions" of the "underlying shape". The expression $j-i+k T$ on the right hand side of Borodin's identity may be understood as the "cylindric hook length" of the box with "cylindric inversion coordinates" $(i, j, k)$ (see section 2).

The right hand side of Borodin's identity may be interpreted combinatorially as a sum over pairs $(\gamma, \mathfrak{A})$ where $\gamma$ is an integer partition and $\mathfrak{A}$ is an arbitrarily labelled "cylindric diagram". The weight of $\mathfrak{A}$ is a sum over the boxes of $\mathfrak{A}$ of the label of the box times the cylindric hook length of the box. The weight of the pair $(\gamma, \mathfrak{A})$ is $T|\gamma|+|\mathfrak{A}|$.

The first result of this paper is a bijective proof of Borodin's identity:

Theorem 1.1 . There exists a natural weight preserving bijection between cylindric plane partitions with given profile $\pi$ and pairs $(\gamma, \mathfrak{A})$ where $\gamma$ is an integer partition and $\mathfrak{A}$ is an arbitrarily labelled cylindric diagram with profile $\pi$.

The proof uses the idea of local rules and growth diagrams first introduced by Fomin [Fom86, Fom95]. The reverse plane partition version of this proof has been previously given by Krattenthaler [Kra06].

Macdonald polynomials are a natural $(q, t)$-deformation of the classical Schur polynomials. The Pieri rules for Macdonald polynomials are very similar to those for the Schur polynomials, only in the Macdonald case certain coefficients appear ([Mac95] page 341).

$$
\begin{aligned}
& \varphi_{\lambda / \mu}(q, t)=\prod_{s \in C_{\lambda / \mu}} \frac{1-q^{a_{\lambda}(s)+1} t^{\ell_{\lambda}(s)}}{1-q^{a_{\lambda}(s)} t^{\ell_{\lambda}(s)+1}} \prod_{s \in C_{\lambda / \mu}} \frac{1-q^{a_{\mu}(s)} t^{\ell_{\mu}(s)+1}}{1-q^{a_{\mu}(s)+1} t^{\ell_{\mu}(s)}} \\
& \psi_{\lambda / \mu}(q, t)=\prod_{s \notin C_{\lambda / \mu}} \frac{1-q^{a_{\lambda}(s)+1} t^{\ell_{\lambda}(s)}}{1-q^{a_{\lambda}(s)} t^{\ell_{\lambda}(s)+1}} \prod_{s \notin C_{\lambda / \mu}} \frac{1-q^{a_{\mu}(s)} t^{\ell_{\mu}(s)+1}}{1-q^{a_{\mu}(s)+1} t^{\ell_{\mu}(s)}}
\end{aligned}
$$

Here $C_{\lambda / \mu}$ denotes the set of columns of $\lambda$ which are longer than the corresponding columns of $\mu$. For any box $s$ define $a_{\lambda}(s)$ to be the "arm length" of $s$ and $\ell_{\lambda}(s)$ to be the "leg length" of $s$ (see section 2).

By using Macdonald polynomials instead of Schur functions, Okada [Oka10] obtained the following $(q, t)$-deformation of Stanley's result:

$$
\sum_{\mathfrak{c} \in \operatorname{RPP}(\pi)} W_{\mathfrak{c}}(q, t) z^{|\mathfrak{c}|}=\prod_{\substack{i<j \\ \pi_{i}>\pi_{j}}} \frac{\left(t z^{j-i} ; q\right)_{\infty}}{\left(z^{j-i} ; q\right)_{\infty}}
$$


Note that we are making use of the hypergoemetric notation: $(a ; q)_{\infty}=\prod_{n \geq 0}\left(1-a q^{n}\right)$. If $\mathfrak{c}=$ $\left(\mu^{0}, \mu^{1}, \ldots \mu^{T}\right)$ then the weight function is given by:

$$
W_{\mathfrak{c}}(q, t)=\prod_{\substack{k=1 \\ \pi_{k}=1}}^{T} \varphi_{\mu^{k} / \mu^{k-1}}(q, t) \prod_{\substack{k=1 \\ \pi_{k}=0}}^{T} \psi_{\mu^{k-1} / \mu^{k}}(q, t)
$$

Observe that when $q=t$, Okada's formula reduces to that of Stanley. The regular plane partition case of Okada's identity had been previously given by Vuletic [Vul09]. The second result of this paper is an analogous $(q, t)$-deformation of Borodin and Tingley's formula for the enumeration of cylindric plane partitions:

\section{Theorem 1.2}

$$
\sum_{\mathfrak{c} \in C P P(\pi)} W_{\mathfrak{c}}(q, t) z^{\mid \mathfrak{c |}}=\prod_{n \geq 0}\left(\frac{1}{1-z^{n T}} \prod_{\substack{i<j \\ \pi_{i}>\pi_{j}}} \frac{\left(t z^{j-i+n T} ; q\right)_{\infty}}{\left(z^{j-i+n T} ; q\right)_{\infty}} \prod_{\substack{i>j \\ \pi_{i}>\pi_{j}}} \frac{\left(t z^{j-i+(n+1) T} ; q\right)_{\infty}}{\left(z^{j-i+(n+1) T} ; q\right)_{\infty}}\right)
$$

The weight function is exactly the same as that given by Okada (equation 10 . When $q=t$ one finds that Theorem (1.2) reduces to equation (6). The proof of Theorem (1.2) uses commutation relations for certain $(q, t)$-vertex operators acting on Macdonald polynomials which are essentially due to Garsia, Haiman and Tesler [GHT99]. The Hall-Littlewood case $(q=0)$ of Theorem $(1.2)$ has been previously given in Corteel, Savelief and Vuletic [CSV11].

Recall that in the plethystic notation [GHT99], if $a(q, t)=\sum_{n, m} a_{n, m} q^{n} t^{m}$ with $a_{n, m} \in \mathbb{Z}$ and $a_{0,0}=0$, then we have:

$$
\Omega[a(q, t)]=\prod_{n, m} \frac{1}{\left(1-q^{n} t^{m}\right)^{a_{n, m}}}
$$

Making use of this notation, the cylindric weight function may be given an explicit combinatorial description which simplifies greatly that given by Okada [Oka10]:

\section{Theorem 1.3}

$$
W_{\mathfrak{c}}(q, t)=\Omega\left[(q-t) \mathcal{D}_{\mathfrak{c}}(q, t)\right]
$$

where the alphabet $\mathcal{D}_{\mathfrak{c}}(q, t)$ is given by:

$$
\mathcal{D}_{\mathfrak{c}}(q, t)=\sum_{s \in \text { peak }(\mathfrak{c})} q^{a_{\mathfrak{c}}(s)} t^{\ell_{\mathfrak{c}}(s)}-\sum_{s \in \operatorname{valley}(\mathfrak{c})} q^{a_{\mathfrak{c}}(s)} t^{\ell_{\mathfrak{c}}(s)}
$$

The precise definition of "valley" and "peak" cubes will be made clear in section 5 . The outline of this paper is as follows. In section 2 we clarify several definitions. In section 3 we discuss the main ideas in the proof of theorem 1.1 In section 4 we recall some basic facts about Macdonald polynomials and then give the key ideas of the proof of Theorem 1.2 Finally in section 5 we introduce the non-intersecting lattice path model for cylindric plane partitions which is necessary in order to define properly the combinatorial formula for the weight function in theorem 1.3 


\section{Definitions}

An integer partition is simply a weakly decreasing list of non-negative integers. If the sum of the parts of $\lambda$ is equal to $n$, then we say that $\lambda$ is a partition of $n$ and write $|\lambda|=n$. The conjugate of the integer partition $\lambda=\left(\lambda_{1}, \lambda_{2}, \ldots, \lambda_{k}\right)$ is defined to be $\lambda^{\prime}=\left(\lambda_{1}^{\prime}, \lambda_{2}^{\prime}, \ldots \lambda_{r}^{\prime}\right)$ where $\lambda_{j}^{\prime}=\#\left\{i \mid \lambda_{i} \geq j\right\}$.

It is often convenient to represent an integer partition visually as a young diagram, which is a collection of boxes in the cartesian plane which are "stacked up" in the bottom right hand corner. Note that our convention differs from both the standard french and english conventions. The reason for this will become clear shortly. The minimum profile of an integer partition is the binary string which traces out the "jaggard boundary" of the associated young diagram. Reading from the top right hand corner to the bottom left hand corner, a zero is recorded for every vertical step and a one for every horizontal step. For example the minimum profile of our example partition $\lambda=(5,3,3,2)$ is 110100110 :

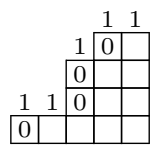

The minimum profile of an integer partition necessarily starts with a one and ends with a zero. An integer partition is uniquely determined by its minimum profile. A generalized profile is an arbitrary string of zeros and ones. Each generalized profile associated to a minimum profile, and hence an integer partition, by removing the leading zeros and trailing ones.

An inversion in a binary string $\pi$ is a pair of indices $(i, j)$ such that $i<j$ and $\pi_{i}>\pi_{j}$. There is a natural bijection between the "boxes" of an integer partition $\lambda$ and the inversions in any generalized profile of $\lambda$. The arm length of the box $s$ of the partition $\lambda$ with "cartesian coordinates" $(i, j)$ is given by $a_{\lambda}(s)=\lambda_{i}-j$. If the box $s$ has "inversion coordinates" $(i, j)$ then the arm length is given by $a_{\lambda}(s)=\#\left\{i<k<j \mid \pi_{k}=1\right\}$. The leg length of the box $s$ of the partition $\lambda$ with "cartesian coordinates" $(i, j)$ is given by $\ell_{\lambda}(s)=\lambda_{j}^{\prime}-i$. If the box $s$ has "inversion coordinates" $(i, j)$ then the arm length is given by $\ell_{\lambda}(s)=\#\left\{i<k<j \mid \pi_{k}=0\right\}$. The hook length of the box $s$ is given by $h_{\lambda}(s)=a_{\lambda}(s)+b_{\lambda}(s)+1$.

We say that $\mu \subseteq \lambda$ if and only if $\mu_{i} \leq \lambda_{i}$ for all $i$. For any pair of partitions $\lambda$ and $\mu$ satisfying $\mu \subseteq \lambda$ we say that $\lambda / \mu$ is a horizontal strip and write $\mu \preceq \lambda$ if and only if for each $j$ we have $\lambda_{j}^{\prime}-\mu_{j}^{\prime} \in\{0,1\}$. In terms of profiles, $\lambda / \mu$ is a horizontal strip if and only if the difference between the position of the $j$-th one in the profile of $\lambda$ and the position of the $j$-th one in the profile of $\mu$ is equal to zero or one.

The "interlacing sequence" definition of a cylindric plane partition has already been given in the introduction. A cube of a cylindric partition $\mathfrak{c}=\left(\mu_{0}, \mu_{1}, \ldots \mu_{T}\right)$ is a box of any of the partitions $\mu_{1}, \mu_{2}, \ldots \mu_{T}$. Note that to avoid double counting we do not include the boxes in the partition $\mu_{0}$.

Cylindric plane partitions are often represented as certain labelled "cylindric diagrams". For example, the cylindric plane partition $\mathfrak{c}=((3,2,2),(5,3,2),(6,4,3,2),(4,3,2),(4,3,2,1),(3,2,2))$ with profile 10100 may be represented as:

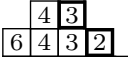

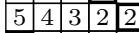

$$
\begin{aligned}
& \begin{array}{lll|l|l}
\hline 3 & 3 & 3 & 2 & 1 \\
\hline
\end{array} \\
& \begin{array}{llll}
2 & 2 & 2 & 0 \\
\hline
\end{array}
\end{aligned}
$$

The individual partitions in the interlacing sequence picture are read off the diagonals. One can imagine "wrapping" this diagram around a cylinder by identifying the first and last diagonals. Equivalently one 
can think of a cylindric diagram as the "fundamental domain" of an infinite young diagram with periodic profile.

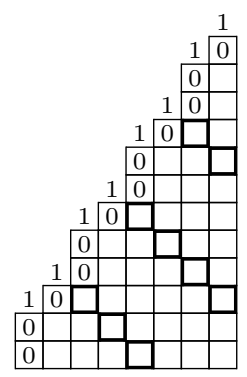

It is natural to index the boxes of the cylindric diagram via "cylindric inversion coordinates" $(i, j, k)$ where $\pi_{i}=1, \pi_{j}=0$ and if $j<i$ then $k \geq 1$ otherwise $k \geq 0$. Here are the cylindric inversion coordinates of each box of our example cylindric diagram:

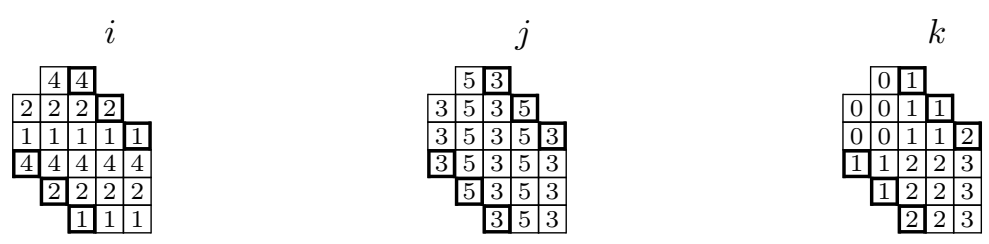

Two boxes lie in the same "cylindric row" if they have the same $i$-coordinate, and in the same "cylindric column" if they have the same $j$-coordinate. The $k$-coordinate is a sort of "depth" or "winding number". The horizontal strip condition for the diagonals implies that the labels must be weakly decreasing along both cylindric rows and cylindric columns. The following definition is crucial for understanding the analogy between cylindric plane partitions and reverse plane partitions:

Definition 2.1 The cylindric hook length of a box with cylindric inversion coordinates $(i, j, k)$ is given by $j-i+k T$.

\section{Theorem 1.1}

The well known RSK correspondence gives a bijection between pairs of semistandard young tableau with the same shape and matrices with non-negative entries. The idea of a growth diagram was first introduced by Fomin [Fom86, Fom95]. Krattenthaler [Kra06] made use of this framework to give a new bijective proof of Stanley's identity (equation 5 .

To motivate the definition of a local rule, consider now the following inductive proof of Cauchy's identity.

Proposition 3.1 .

$$
\sum_{\lambda} S_{\lambda}\left[X_{n}\right] S_{\lambda}\left[Y_{n}\right]=\prod_{i, j=1}^{n} \frac{1}{1-x_{i} y_{j}}
$$

Proof: By the Pieri formula (equation 3), and the induction assumption, we have on the right hand side: 


$$
\begin{aligned}
R H S(n) & =\left(\sum_{r} h_{r}\left[X_{n-1}\right] x_{n}^{r}\right)\left(\sum_{s} h_{s}\left[Y_{n-1}\right] x_{n}^{s}\right)\left(\sum_{\mu} S_{\mu}\left[X_{n-1}\right] S_{\mu}\left[Y_{n-1}\right]\right) \frac{1}{1-x_{n} y_{n}} \\
& =\sum_{\alpha, \beta} S_{\alpha}\left[X_{n-1}\right] S_{\beta}\left[Y_{n-1}\right]\left(\frac{1}{1-x_{n} y_{n}} \sum_{\mu \in D(\alpha) \cap D(\beta)} x_{n}^{|\alpha / \mu|} y_{n}^{|\beta / \mu|}\right)
\end{aligned}
$$

while by the dual Pieri formula (equation 4), we have on the left hand side:

$$
\begin{aligned}
\operatorname{LHS}(n) & =\sum_{\lambda} S_{\lambda}\left[X_{n-1}+x_{n}\right] S_{\lambda}\left[X_{n-1}+y_{n}\right] \\
& =\sum_{\alpha, \beta} S_{\alpha}\left[X_{n-1}\right] S_{\beta}\left[Y_{n-1}\right]\left(\sum_{\lambda \in U(\alpha) \cap U(\beta)} x_{n}^{|\lambda / \alpha|} y_{n}^{|\lambda / \beta|}\right)
\end{aligned}
$$

In order to complete this proof, we need, for any given $\alpha$ and $\beta$ a bijection between the set of pairs $(m, \mu)$ with $\mu \in D(\alpha) \cap D(\beta)$ and $m$ a non-negative integer and the set of partitions $\lambda \in U(\alpha) \cap U(\beta)$ in such a way that $|\lambda|=|\alpha|+|\beta|-|\mu|+m$. A local rule is just such a bijection.

$$
\mathfrak{L}_{\alpha, \beta}:\left(\mathbb{Z}_{\geq 0}, D(\alpha) \cap D(\beta)\right) \rightarrow U(\alpha) \cap U(\beta)
$$

There are several possible choices of local rule, we shall not describe any of them explicitely here, but rather refer the reader to [Ada08] or [Kra06]. When working with growth models [Fom86, Fom95] it helps to think of the underlying shape of a reverse plane partition as a subset of the lattice $\mathbb{Z}^{2}$, with the "vertices" inheriting a natural poset structure. In a similar spirit, the "underlying shape" of a cylindric plane partition $\mathfrak{c}=\left(\mu^{0}, \mu^{1}, \ldots, \mu^{T}\right)$, which we shall henceforth refer to as a cylindric diagram, may be interpreted as a subset of the lattice $\mathbb{Z}^{T}$ modulo the equivalence relation:

$$
(x, y)=(x+n, y-m)
$$

where $n$ is the number of zeros in the profile of $\mathfrak{c}$ and $m$ is the number of ones. The vertices of a cylindric diagram thus inherit a poset structure.

Let us write $v \triangleleft w$ to indicate that the vertex $v$ is connected to the edge of the vertex $w$ with $v$ below or to the right of $w$. A cylindric growth diagram is a cylindric diagram whose vertices are labelled by integer partitions and whose boxes are labelled by non-negative integers in such that the following conditions are satisfied:

- If $v \triangleleft w$, and if $\lambda$ is the integer partition labelling the vertex $v$ and $\mu$ is the integer partition labelling the vertex $w$, then $\lambda / \mu$ is a horizontal strip.

- All the vertices along the lower boundary have the same label $\gamma$. 
- If $u, v 1, v 2$ and $w$ are four vertices forming a box with label $m$, with $u \triangleleft v 1 \triangleleft w$ and $u \triangleleft v 2 \triangleleft w$, and if the labels of $u, v 1, v 2$ and $w$ are $\mu, \alpha, \beta$ and $\lambda$ respectively then $\mathfrak{L}_{\alpha, \beta}(m, \mu)=\lambda$.

Here is an example (rotated 45 degrees):

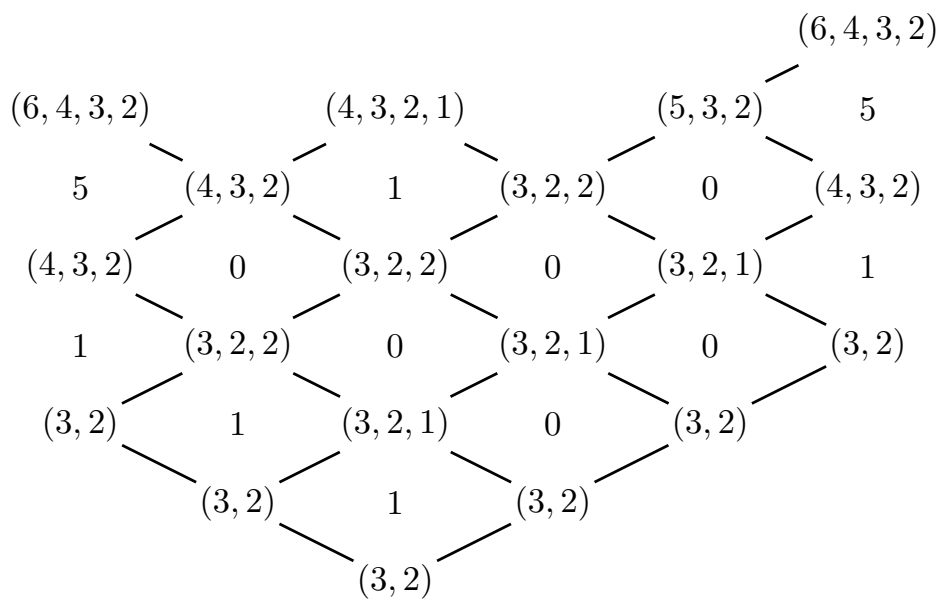

Observe that the sequence of partitions labelling the vertices along the upper boundary forms a cylindric plane partition. Furthermore, once the labels on the upper boundary are fixed, the compatibility condition with the local rule ensures that there is a unique manner to label the remaining boxes and vertices. Conversely, if the labels on the lower boundary are fixed, then the boxes may be labelled in an arbitrary fashion. Once this has been done, there is a unique way to fill in the rest of the labels of the vertices.

Proposition 3.2 For any cylindric growth diagram, the sum of weights of the integer partitions labelling the vertices of the lower boundary, plus the sum over the boxes of the label times the cylindric hook length of the boxes, is equal to the sum of the weights of the integer partition labelling the vertices of the upper boundary.

Proof: To appear in a longer version of this paper.

By passing from a cylindric plane partition to its associated cylindric growth diagram, and then forgetting the labels on all the vertices except those along the bottom boundary, one may effectively construct a weight preserving bijection between the left hand side and the right hand side of Borodin's identity.

\section{Theorem 1.2}

We sketch only the main ideas of the proof. The details will appear in a longer version of this paper.

Let $\Lambda_{q, t}$ denote the ring of symmetric functions over the field of rational functions in the indeterminants $q$ and $t$, and let $\langle-,-\rangle_{q, t}$ denote the Macdonald inner product ([Mac95] page 309). The Macdonald polynomials $\left\{P_{\lambda}(X ; q, t)\right\}$ are an orthogonal, but not orthonormal, basis with respect to this inner product (Mac95] page 322). The dual basis is denoted by $\left\{Q_{\lambda}(X ; q, t)\right\}$. 
The operator $\Omega_{q, t}[X z]$ is defined by:

$$
\Omega_{q, t}[X z]=\prod_{i} \frac{\left(t x_{i} z ; q\right)_{\infty}}{\left(q x_{i} z ; q\right)_{\infty}}
$$

The adjoint of this operator is denoted by $\Omega_{q, t}^{*}[X z]$. The Pieri formulae for the Macdonald polynomials ([Mac95] (page 340 - 341) may be expressed in the form:

$$
\begin{aligned}
\Omega[X z]_{q, t} P_{\mu}(X ; q, t) & =\sum_{\lambda \in U(\mu)} \varphi_{\lambda / \mu}(q, t) P_{\lambda}(X ; q, t) z^{|\lambda|-|\mu|} \\
\Omega^{*}[X z]_{q, t} P_{\lambda}(X ; q, t) & =\sum_{\mu \in D(\lambda)} \psi_{\lambda / \mu}(q, t) P_{\mu}(X ; q, t) z^{|\lambda|-|\mu|}
\end{aligned}
$$

where $U(\mu)$ is the set of partitions which can be obtained from $\mu$ by adding a horizontal strip, $D(\lambda)$ denotes the set of all partitions which can be obtained from $\mu$ by removing a horizontal strip, and $\varphi_{\lambda / \mu}(q, t)$ and $\psi_{\lambda / \mu}(q, t)$ are defined in equations 7 and 8 .

The following lemma is crucial for the proof of Theorem 1.2

\section{Lemma 4.1 (Garsia, Haiman, Tesler [GHT99])}

$$
\Omega_{q, t}^{*}[X u] \Omega_{q, t}[X v]=\prod_{n \geq 0}\left(\frac{1-t q^{n} u v}{1-q^{n} u v}\right) \Omega_{q, t}[X v] \Omega_{q, t}^{*}[X u]
$$

Let $D_{z}$ denote the "degree" operator:

$$
D_{z} P_{\lambda}[X ; q, t]=z^{|\lambda|} P_{\lambda}[X ; q, t]
$$

One can easily check that $D_{z} \Omega_{q, t}[X]=\Omega_{q, t}[X z] D_{z}$ as well as $D_{z} \Omega_{q, z}^{*}[X]=\Omega_{q, t}^{*}\left[X z^{-1}\right] D_{z}$. Fix a profile $\pi$. As a direct consequence of the Pieri formulae (equations 18 and 19 , we have:

$$
\sum_{\mathfrak{c} \in C P P(\pi)} W_{\mathfrak{c}}(q, t) z^{|\mathfrak{c}|}=\sum_{\lambda}\left\langle Q_{\lambda}[X] \mid D_{z} G^{\pi_{0}}[X] D_{z} G^{\pi_{1}}[X] \cdots D_{z} G^{\pi_{T-1}}[X] P_{\lambda}[X]\right\rangle_{q, t}
$$

where $G^{0}[X]=\Omega_{q, t}[X]$ and $G^{1}[X]=\Omega_{q, t}^{*}[X]$. The "trick" now is to repeatedly apply Lemma 4.1 . pulling out terms in the product on the right hand side as we do so. The new feature in the cylindric case is that we must make use of the fact that for any $\left\{u_{i}\right\}_{i \in I}$ and $\left\{w_{j}\right\}_{j \in J}$ we have: 


$$
\begin{aligned}
& \sum_{\mu}\left\langle Q_{\mu}[X] \mid \prod_{i \in I} \Omega_{q, t}\left[X u_{i}\right] \prod_{j \in J} \Omega_{q, t}^{*}\left[X w_{j}^{-1}\right] D_{z}^{T} P_{\mu}[X]\right\rangle_{q, t} \\
& =\sum_{\lambda, \mu}\left\langle Q_{\mu}[X] \mid \prod_{i \in I} \Omega_{q, t}\left[X u_{i}\right] P_{\lambda}[X]\right\rangle_{q, t}\left\langle Q_{\lambda}[X] \mid \prod_{j \in J} \Omega_{q, t}^{*}\left[X w_{j}^{-1}\right] D_{z}^{T} P_{\mu}[X]\right\rangle_{q, t} \\
& =\sum_{\lambda, \mu}\left\langle Q_{\lambda}[X] \mid \prod_{j \in J} \Omega_{q, t}^{*}\left[X w_{j}^{-1}\right] D_{z}^{T} P_{\mu}[X]\right\rangle_{q, t}\left\langle Q_{\mu}[X] \mid \prod_{i \in I} \Omega_{q, t}\left[X u_{i}\right] P_{\lambda}[X]\right\rangle_{q, t} \\
& =\sum_{\lambda}\left\langle Q_{\lambda}[X] \mid \prod_{j \in J} \Omega_{q, t}^{*}\left[X w_{j}^{-1}\right] D_{z}^{T} \prod_{i \in I} \Omega_{q, t}\left[X u_{i}\right] P_{\lambda}[X]\right\rangle \\
& =\sum_{\lambda}\left\langle Q_{\lambda}[X] \mid \prod_{j \in J} \Omega_{q, t}^{*}\left[X w_{j}^{-1}\right] \prod_{i \in I} \Omega_{q, t}\left[X u_{i} z^{T}\right] D_{z}^{T} P_{\lambda}[X]\right\rangle
\end{aligned}
$$

As well as the fact that:

$$
\begin{aligned}
& \lim _{k \rightarrow \infty} \sum_{\lambda}\left\langle Q_{\lambda}[X] \mid \prod_{j \in J} \Omega_{q, t}^{*}\left[X w_{j}^{-1}\right] \prod_{i \in I} \Omega_{q, t}\left[X u_{i} z^{k T}\right] D_{z}^{T} P_{\lambda}[X]\right\rangle \\
& =\sum_{\lambda}\left\langle Q_{\lambda}[X] \mid D_{z}^{T} P_{\lambda}[X]\right\rangle \\
& =\prod_{n} \frac{1}{1-z^{n T}}
\end{aligned}
$$

It is perhaps worth remarking that the nature of the proof is such that the identity remains true if on the left hand side we replace:

$$
z^{|\mathfrak{c}|} \mapsto z_{0}^{\left|\mu_{0}\right|} z_{1}^{\left|\mu_{1}\right|} \cdots z_{T-1}^{\left|\mu_{T-1}\right|}
$$

while on the right hand side we replace:

$$
\begin{aligned}
z^{n T} & \mapsto z_{0}^{n} z_{1}^{n} \cdots z_{T-1}^{n} & & \\
z^{j-i+n T} & \mapsto z_{0}^{n} z_{1}^{n} \cdots z_{i}^{n} z_{i+1}^{n+1} \cdots z_{j}^{n+1} z_{j+1}^{n} \cdots z_{T-1}^{n} & & \text { when } i<j \\
z^{j-i+(n+1) T} & \mapsto z_{0}^{n+1} z_{1}^{n+1} \cdots z_{j}^{n+1} z_{j+1}^{n}+\cdots z_{i}^{n} z_{i+1}^{n+1} \cdots z_{T-1}^{n+1} & & \text { when } i>j
\end{aligned}
$$

This provides a new refined version of Borodin's identity even in the Schur case. It was previously remarked in the reverse plane partition case by [Oka10].

\section{Theorem 1.3}

In this section we define a bijection between cylindric plane partitions and non-intersecting lattice paths on the cylinder. The non-intersecting lattice path model is equivalent to a model of rhombus tilings on a cylinder. We define what we mean by a "peak cube" and a "valley cube" in Theorem 1.3 and we also show how our theorem reduces to the "border strip formula" of [CSV11] when $q=0$.

Let us begin with an example: 

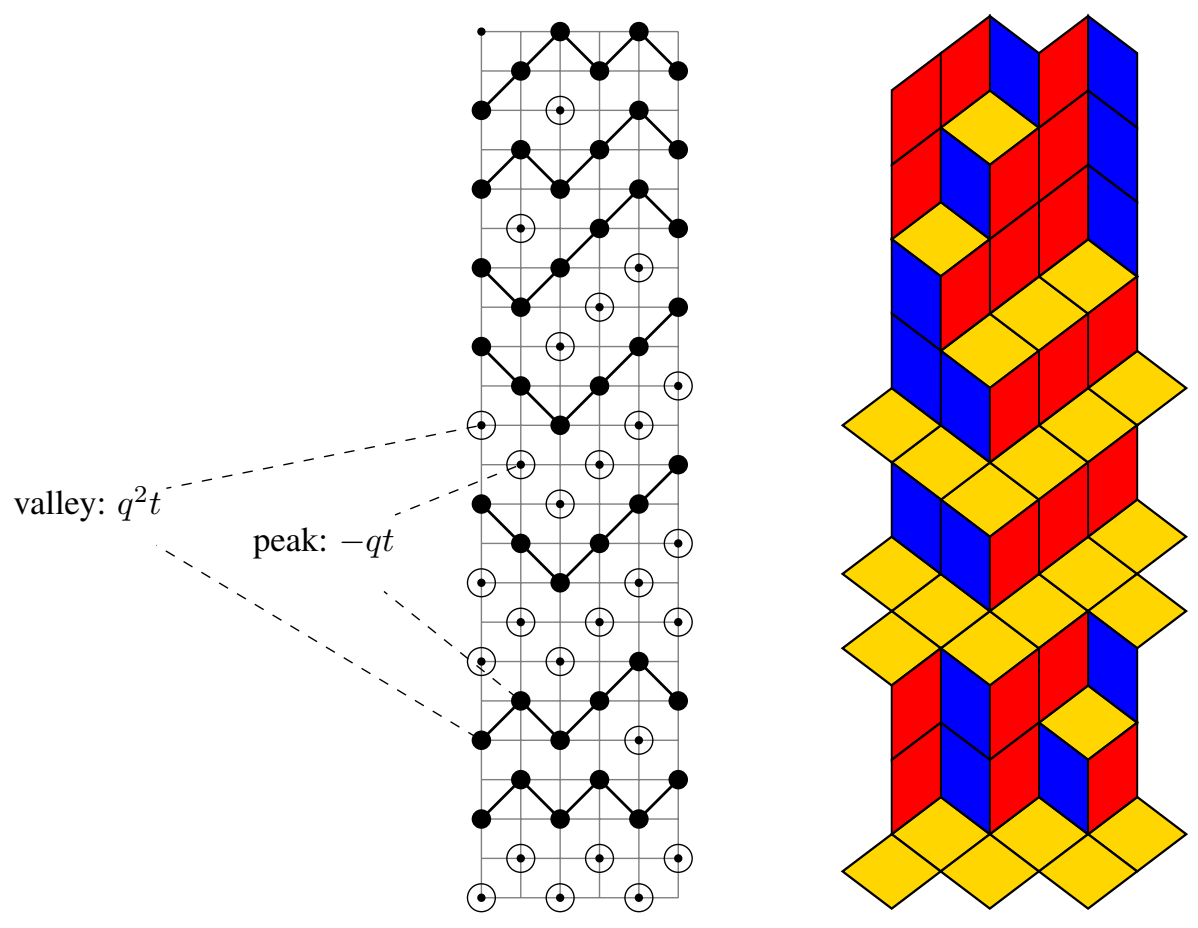

$$
\mathfrak{c}=((3,2,2),(5,3,2),(6,4,3,2),(4,3,2),(4,3,2,1),(3,2,2))
$$

The correspondence between the path model and the tiling model is clear. The yellow tiles correspond to white vertices. The red tiles correspond to up steps of a path. The blue tiles correspond to down steps of a path. If $\mathfrak{c}=\left(\mu^{0}, \mu^{1}, \ldots, \mu^{k}\right)$ then the parts of are given by the "heights" of the yellow tiles in the rhombus tiling model. Equivalently a generalized profile for the partition $\mu^{k}$ may be read off the $k$-th vertical of the non-intersecting path model. Reading from bottom to top, each white vertex corresponds to a zero and each black vertex corresponds to a one (see section 2).

A cube in the non-intersecting lattice path model is a pair of vertices $u=\left(x, y_{1}\right)$ and $v=\left(x, y_{2}\right)$ with $u$ coloured black, $v$ coloured white and $y_{1}<y_{2}$. The arm length of the cube $(u, v)$ is the number of black vertices which appear on the same vertical, strictly between $u$ and $v$. The leg length of the cube $(u, v)$ is the number of white vertices which appear on the same vertical, strictly between $u$ and $v$ (see section 2).

The path associated to the cube $(u, v)$ is the path which passes through the black vertex $v$. A valley cube is a cube $(u, v)$ for which the associated path takes a down step just before passing through $v$, followed immediately by an upstep. A peak cube is a cube $(u, v)$ for which the associated path takes an step just before passing through $v$, followed immediately by a downstep.

We have marked one peak cube and one value cube on the diagram, together with their contribution to the alphabet $\mathfrak{D}_{\mathfrak{c}}(q, t)$ in Theorem 1.3. Don't forget that we are working on a cylinder, so that the first vertical is identified with the last vertical in such a way that each path forms a closed loop.

Finally, let us define a surface cube to be a cube $(u, v)$ such that if $u=\left(x, y_{1}\right)$ and $v=\left(x, y_{2}\right)$ then for all $w=\left(x, y^{\prime}\right)$ with $y_{1}<y^{\prime}<y_{2}$ the vertex $w$ is coloured white. Surface cubes are naturally in 
bijection with yellow tiles in the rhombus tiling model. The level of a surface cube $(u, v)$ is $y_{2}-y_{1}$ where $u=\left(x, y_{1}\right)$ and $v=\left(x, y_{2}\right)$. That is to say, the level of a surface cube is its leg length. With these definition one can see that our combinatorial formula for the cylindric weight function reduces to the border strip formula of [CSV11] when $q=0$.

The proof of Theorem 1.3 involves a careful cancellation of terms appearing in the various Pieri coefficients (equations 7 and 8 which make up the definition of the weight function (equation 10). The details will appear in a longer version of this paper.

\section{References}

[Ada08] Shingo Adachi. Reverse plane partitions and growth diagrams. preprint, 2008.

[Bor07] Alexei Borodin. Periodic Schur process and cylindric partitions. Duke Math. J., 140(3):391468, 2007.

[CSV11] Sylvie Corteel, Cyrille Savelief, and Mirjana Vuletić. Plane overpartitions and cylindric partitions. J. Combin. Theory Ser. A, 118(4):1239-1269, 2011.

[Fom86] S. V. Fomin. The generalized Robinson-Schensted-Knuth correspondence. Zap. Nauchn. Sem. Leningrad. Otdel. Mat. Inst. Steklov. (LOMI), 155(Differentsialnaya Geometriya, Gruppy Li i Mekh. VIII):156-175, 195, 1986.

[Fom95] Sergey Fomin. Schur operators and Knuth correspondences. J. Combin. Theory Ser. A, 72(2):277-292, 1995.

[GHT99] A. M. Garsia, M. Haiman, and G. Tesler. Explicit plethystic formulas for Macdonald $q, t$ Kostka coefficients. Sém. Lothar. Combin., 42:Art. B42m, 45 pp. (electronic), 1999.

[GK97] Ira M. Gessel and C. Krattenthaler. Cylindric partitions. Trans. Amer. Math. Soc., 349(2):429479, 1997.

[Kra06] C. Krattenthaler. Growth diagrams, and increasing and decreasing chains in fillings of Ferrers shapes. Adv. in Appl. Math., 37(3):404-431, 2006.

[Mac95] I. G. Macdonald. Symmetric functions and Hall polynomials. Oxford Mathematical Monographs. The Clarendon Press Oxford University Press, New York, second edition, 1995.

[Oka10] Soichi Okada. $(q, t)$-deformations of multivariate hook product formulae. J. Algebraic Combin., 32(3):399-416, 2010.

[OR03] Andrei Okounkov and Nikolai Reshetikhin. Correlation function of Schur process with application to local geometry of a random 3-dimensional Young diagram. J. Amer. Math. Soc., 16(3):581-603 (electronic), 2003.

[Tin08] Peter Tingley. Three combinatorial models for $\widehat{\mathrm{sl}}_{n}$ crystals, with applications to cylindric plane partitions. Int. Math. Res. Not. IMRN, (2):Art. ID rnm143, 40, 2008.

[Vu109] Mirjana Vuletić. A generalization of MacMahon's formula. Trans. Amer. Math. Soc., 361(5):2789-2804, 2009. 